\title{
SELLING SIMULATION AND SIMULATION RESULTS
}

\author{
Randall P. Sadowski \\ Systems Modeling Corporation \\ The Park Building \\ 504 Beaver Street \\ Sewickley, PA 15143, U.S.A
}

\begin{abstract}
Selling simulation as a valid analysis tool is the first, and maybe the most important, step in the simulation process. Once the decision has been made to use simulation, the analyst must then be concerned with selling the ultimate results produced by the simulation.

Most simulation studies are performed by engineers or technical individuals who understand the capabilities and merits of simulation. They also frequently assume that the results of a simulation study will automatically be accepted by management. Given that the system under study has been accurately captured by the simulation and that a thorough analysis has been performed, it seems logical that results will speak for themselves and be accepted. Unfortunately, many decisions are not based solely on logic; and occasionally, results of a valid simulation are discarded by decision makers because they do not fully understand the implications or because they prefer not to believe the outcome. This paper concentrates on the issues regarding the preparation, presentation and selling of the results and attempts to provide guidelines to assure that the information provided by the simulation study is used appropriately.
\end{abstract}

\section{SELLING THE CONCEPT OF SIMULATION}

Any simulation software salesmen can provide dozens of reasons why you should buy their software and use it for simulation analysis. Simulation allows you to draw inferences about new systems without building them or make changes to existing systems without disturbing them. An accurate simulation will allow you to pose a series of "what if" questions and determine the system's response in terms of key performance measures. It is probably the only tool that will allow total system interactions to be analyzed.

Unfortunately, there is a cost associated with simulation. In addition to the software expense, there is the analyst's time and the additional time required by the project team to provide the essential information. The key question that must be answered is: Do the benefits equal or exceed the costs? In most cases, the answer is yes. However, the actual benefits are often very difficult, if not impossible, to quantify whereas the costs are very easy to identify.

There have been numerous occurrences where corporations have created large, successful simulation groups only to disband them in an attempt to cut costs. The primary reason given is that such a group adds no direct value and that the associated costs cannot be justified. The dilemma is: How do you place a value on building or modifying a system right the first time? One could occasionally not simulate a project that has high visibility, high cost, and high probability of failure; and then after the project has failed, use simulation to improve the system and show what costs could have been saved if the project had been simulated at the start. Although this is probably more an act of desperation and obviously not a solution to justifying the use of simulation, it does illustrate the problems faced by simulation advocates. A better approach is to admit that one cannot define the benefits fully and then accept the fact that there is tremendous merit in doing it right the first time.

\section{STARTING THE SIMULATION STUDY}

When one thinks of selling simulation and simulation results, typically one thinks in terms of the carpeted board room and the final presentation. However, if the results of a simulation study are to be accepted, the selling must begin at the very start of the simulation project. The final presentation is only the last step in the process. The long-term success of simulation is dependent upon the quality of results obtained for the current studies as well as those from the past. A decision maker who was assured that a previous system would work because it was simulated and subsequently found that the system turned out to be a disaster is liable to be 
very skeptical of believing and following simulation results.

Thus, in leading up to the issues surrounding the final presentation, some assumptions must be made about the validity of the results. Valid results start at the very beginning of a simulation study. One must completely understand the perceived or real problem that caused the study to be performed. The objectives should be clearly stated and accepted by everyone involved. This could include a broad range of individuals, including shop floor staff, design and process engineers, equipment and system contractors as well as the ultimate decision makers. In reality, the selling starts at this point in the project. Ideally, one should understand the role that each of these individuals might choose to play in the overall project. Each individual may have different concerns (and often hidden agendas) that the analysts will have to consider at some point in the project. The sooner these concerns and agendas are addressed, the better.

The validity of the project may hinge upon doing a successful job of selling the merits of the simulation study to those involved in designing and operating the system, since you will depend on them for information, insight, and honest assessment of the planned or modified system. Reluctant participants are likely to be less willing, or careful, in providing information about the system. Even worse, they may purposefully give incorrect information in an attempt to torpedo the simulation project. Willing and eager participants are inclined to play a major role and will use the resulting simulation as a tool to better understand their proposed system.

Once the objectives have been clearly stated, the system description must be developed and the level of detail that will be included in the model must be determined. Then the model development can be initiated. We will assume that these steps are undertaken by experienced analysts and that the resulting model is an accurate representation of the system under study.

\section{ESTABLISHING MODEL CREDIBILITY}

Given an accurate system description, the actual simulation model can be developed. Next the analyst must establish the credibility of the model with the project team and the major players who understand the system under study by proving that the simulation does behave like the proposed system. This would include shop floor staff, engineers, and possibly contractors. The intent is to show these players that the model does, in fact, capture the system interactions and behave like the system under study. This task is much easier if the study concerns an existing system. With an existing system, it is possible to compare directly the real and the simulation results. Specific scenarios that have occurred in the real system can be created in the model to show that it reacts in the same manner. If the system under study is one that is proposed, then this task is more difficult as a direct comparison is not possible. If a similar system exists, an aggregate comparison can be made. Otherwise, credibility must be established by observing the manner in which the system operates. This is best accomplished with animation. This may be the same animation used during verification and need not be elaborate. A simple display showing equipment status and parts moving through the system is often adequate for individuals familiar with the study system.

Different players will tend to concentrate on different aspects of the system. Engineers will be more interested in specific equipment or overall system logic. Shop floor staff will most likely concentrate on system activities for which they have responsibility. Contractors will be more concerned with the pieces of the system for which they are supplying equipment or logic. Different animations may be required, but they can be simplistic in form as they only need to illustrate how the system operates.

Establishing model credibility with this group of players is a critical step in the process as their support of the results from the model can easily mean the difference between success and failure. An excellent formula for success is a united team that believes in and supports the modeling effort.

\section{PERFORMING THE ANALYSIS}

The type and depth of analysis that needs to be performed depends on the initial objectives. Although each study probably requires a unique approach, for this paper let us assume that there are three basic categories of problems/objectives. The first is a new or modified system for which the primary objective is to determine whether or not the designed system will work. This is perhaps the easiest problem in that it only requires a "yes" or "no" answer. If the system performs as expected after the first round of analysis, the simulation effort may be completed. If the system does not perform as expected, it may fall into the second category. In this category, there is often an existing system that is not performing as expected, and the objective is to determine what the problem is and then figure out how to make it work better. The third category deals with the development of a new system where the primary objective is to establish a proof of concept and to estimate system performance. This last type is different from the first in that there may not be a specific system design, but only general concepts. The objective of the simulation project is to develop a workable system design using these concepts. 
Although these categories may seem quite similar, the level of experience, creativity, and/or expertise required to perform a good analysis is much higher for the last two categories. You must first determine why the system is not functioning at the desired level, then you must determine what needs to be changed to reach that level. These changes could be in the resources, control logic, or production mix areas. Before going forward with what appears to be the obvious solution, check it for validity. For example, the solution may require an additional machine in order to relieve the primary bottleneck. However, if floor space is not available to place the machine or if funds are not available to buy the machine, you have not arrived at a feasible solution.

During the analysis, we will assume that good statistical practices have been followed. It is assumed that issues such as steady state and length of run have been addressed and that the resulting answers are presented as confidence intervals rather than point estimates. Furthermore, we will assume that no observations are made on system capabilities that have not been explicitly modeled.

\section{THE FINAL PRESENTATION}

Once the analysis has been completed and the objectives of the study have been met, it is time to present the results to the decision makers. This might require making a formal presentation or a written report, or both. In any case, it is important to understand the audience and their expectations. Most decision makers would prefer a simple "yes" or "no" conclusion with a few supporting numbers. For example, yes, the new system will function as predicted, and it will yield a stated throughput. However, if the results are not what the decision makers expected, often they will want a very detailed explanation of why the results are different from what was projected. When this occurs, it is important that the analysts can present information in a format that the decision makers can easily understand.

\subsection{Illustrating the Results}

In addition to the overall study conclusions, the analyst should be prepared to illustrate the results. There are basically two ways to accomplish this: animation and data. Animation is best used to allow the decision makers to see their system operate as it was predicted by the simulation under a variety of preselected conditions. It is very useful in illustrating system problems or highlighting the causes of those problems. In the early phases of the study, the animations may have looked like schematics of the system. Since the earlier players were generally very familiar with the system, detailed or realistic animations were not required. Prior to the final presentation, it may be wise to spend additional time to create a more realistic animation of the system. Generally speaking, the higher the decision makers are in the organization, the more desirable it is to have a realistic animation. The exception might be where these individuals have retained a high and current degree of technical capability. Providing an animation that allows the decision makers to view and easily understand the positive or negative aspects of the results can often be the deciding factor in their acceptance of the results.

\subsection{Presenting the Data}

Although an animation can often be used as the primary selling tool, the proper presentation and interpretation of data that describes the system's performance is equally important. Try to avoid giving definitive point answers for system performance. In most cases, the results from the simulation are based on estimates of operational data. Rarely is this data truly accurate, and often it is only an educated guess. One should identify which data is questionable and perform sensitivity analysis to determine how responsive the system performance measures are to the data.

To illustrate this point, let us assume that the failure times for new equipment in the study system are determined to be questionable. Make several simulation runs for a range of different failure times to determine the system's reaction. If the system performance is very dependent upon these estimates, then the resulting information should be presented to illustrate this dependency. For example, a plot of system performance versus percent of failure would illustrate this point. It is also suggested that you select a confidence level and plot the confidence bands along with the mean estimates to emphasize the potential variability in the system. This type of data presentation illustrates not only the data interaction in the system, but also the variability of the expected performance.

Another useful method for displaying simulation results is the time series plot. For example, the average inventory level at a workcenter may be very misleading. The actual level may fluctuate over a wide range. A time series plot will show this variability and also indicate how often the level reaches the extremes. This type of plot can be used to display any system performance measure that tends to vary over a wide range. 


\subsection{The Presentation Format}

If a detailed simulation analysis is performed, the analysts can accumulate massive amounts of data. The tendency is to try to present all this information. The result is often information overload. Or even worse, a manager will zero in on a single piece of data or information that is not really important. A better approach is to prepare all the information, but only present the visual picture accompanied by the key results. However, the remaining information will be available if specific questions arise during the presentation. Generally, one should try to anticipate the direction the questions will take and have visuals prepared in order to be able to respond immediately.

One final point that should always be considered is the simple fact that the results from a simulation are rarely 100 percent accurate. Remember that the simulation model is an abstraction of the actual system, and typically the data that drives the model are estimates. Therefore, in presenting the results, one should always attempt to quantify the risks as well as the benefits.

\section{CONCLUSION}

The acceptance of simulation results by an organization is definitely dependent upon the history of simulation in that organization. Successful past projects that were simulated are remembered, but rarely is credit given to the simulation study for the overall project success. Often the attitude is, "Sure, the simulation told us it would work, but we knew that before we simulated it." This attitude often persists even if the results of the simulation caused significant changes to be made. Also, the good results are quickly forgotten as the project team and managers move on to their next task.

However, a disastrous project that was simulated is remembered for a long, long time. Even if the simulation was not at fault, the tendency is to assume that it did not work properly for that project. Thus, the good name of simulation established by 100 successful projects can be canceled by one major disaster.

One should remember that it is important to sell throughout the entire simulation project, not just at the final presentation. This approach will often maximize the input by all members of the project team and increase the likelihood of a success.

\section{REMARKS}

This article was previously published in the 1992 Winter Simulation Conference Proceedings and has been edited for resubmission in 1993.

\section{AUTHOR BIOGRAPHY}

RANDALL P. SADOWSKI is currently vice president of Systems Modeling Corporation in charge of consulting and user-education services. He was previously on the faculty at Purdue University and at the University of Massachusetts. He received his bachelors and masters degrees from Ohio University and his Ph.D. from Purdue. Dr. Sadowski's research interests are in manufacturing and production systems with emphasis on modeling, control, and applied scheduling. He has authored numerous technical articles and papers, served as chairman of the Third International Conference of Production Research, and was the General Chair of the 1990 Winter Simulation Conference. He is a senior member of the Institute of Industrial Engineers and is serving a two-year term as Vice President of Systems Integration. He also served as editor of a two-year series on Computer Integrated Manufacturing Systems for IE Magazine (which received the 1987 IIE outstanding publication award) and was a co-editor of Computer Integrated Manufacturing Systems: Selected Readings, published by IIE. 